\title{
Electric field induced hydrogenation of silicene
}

Cite this: Phys. Chem. Chem. Phys., 2014, 16, 16588

Received 1st April 2014, Accepted 26th June 2014

DOI: $10.1039 / c 4 c p 01416 b$

www.rsc.org/pccp

\author{
Weichang $\mathrm{Wu}^{\mathrm{a}}$ Zhimin $\mathrm{Ao},{ }^{\text {b }}$ Tao Wang, ${ }^{\mathrm{c}}$ Changming $\mathrm{Li}^{\mathrm{d}}$ and Sean $\mathrm{Li}^{\mathrm{a}}$
}

An alternative approach for hydrogenation of silicene is proposed through applying an external electric field in order to reduce the reaction energy barrier based on density functional theory calculations. It is found that a positive perpendicular electric field $\mathrm{F}$ can act as a catalyst to reduce the energy barrier of $\mathrm{H}_{2}$ dissociative adsorption on silicene, which facilitates the hydrogenation of silicene. In addition, it is found that the barrier decreases as $F$ increases, and when $F$ is above 0.05 a.u. $\left(1\right.$ a.u. $\left.=5.14 \times 10^{11} \mathrm{~V} \mathrm{~m}^{-1}\right)$, the barrier is quite low and hydrogenation of silicene can take place efficiently at room temperature. The catalytic effect of the electric field on hydrogenation of silicene is induced by the redistribution of atomic charge under the electric field, which would change the chemical activity of silicene significantly.

\section{Introduction}

Graphene has a two-dimensional honeycomb structure with unique electronic, mechanical and thermodynamic properties, which has been attracting great attention since it was fabricated in 2004..$^{1-3}$ It is known that intrinsic graphene is a semiconductor with zero band gap, the band gap can be opened and the width of the gap is controllable through different ways. ${ }^{4-7}$ Hydrogenation is one of the effective methods that can be used to control the electronic properties of graphene. ${ }^{8-11}$ For example, graphene can undergo full hydrogenation and become a wide gap semiconductor with a band gap of around $3.5 \mathrm{eV} .^{8}$ The hydrogenated graphene, graphane, is stable at room temperature. ${ }^{12}$ Furthermore, it has been reported that through hydrogenation graphene can be switched from metallic to semiconducting, and from nonmagnetic to magnetic. ${ }^{13}$ These discoveries bring vital perspectives for the possible applications of graphene-based devices. For instance, graphene is found to be a promising material for hydrogen storage with high hydrogen storage capacity and reversible hydrogen storage and release. ${ }^{14,15}$

These remarkable achievements in graphene have resulted in more and more attention being paid to the other elements in the IV group in order to find similar properties. Newly reported silicene, a two-dimensional honeycomb network of silicon atoms, is the hottest one of this type ${ }^{16-20}$ This special silicon

\footnotetext{
${ }^{a}$ School of Materials Science and Engineering, The University of New South Wales, Sydney, NSW 2052, Australia

${ }^{b}$ Centre for Clean Energy Technology, School of Chemistry and Forensic Science, University of Technology, PO Box 123, Broadway, Sydney, NSW 2007, Australia. E-mail: Zhimin.ao@uts.edu.au

${ }^{c}$ College of Electrical Engineering, Zhejiang University, Hangzhou 130027, China

${ }^{d}$ Chongqing Key Lab for Advanced Materials \& Clean Energies of Technologies, Institute for Clean Energy and Advanced Materials, Southwest University,

2 Tiansheng Rd, Beibei, Chongqing, 400715, China
}

component does not exist in nature and was first predicted as an aromatic stage of two-dimensional Si with a buckled structure in 1994 through first-principles calculations. ${ }^{21}$ The authors claimed that there was a linear dispersion around the Dirac cone at the Fermi level at the $K$ point like in graphene. Several years later, a single layer sheet of $\mathrm{Si}$ was obtained on a substrate via the chemical exfoliation of calcium disilicide ${ }^{22}$ and single-wall silicon nanotubes were achieved on $\mathrm{Ag}(110)$ or $\mathrm{Ag}(100)$ substrates. ${ }^{23}$ It is known that the electronic configurations of silicon are similar to those of carbon. However, in silicon the $\mathrm{sp}^{3}$ hybridization is more stable than the $\mathrm{sp}^{2}$ hybridization, ${ }^{24}$ which is in contrast to that of carbon. Therefore, silicon cannot spontaneously form a honeycomb structure as carbon but a low buckled structure instead. Although isolated silicene has not been reported experimentally yet, different theoretical studies have proven that this isolated two-dimensional buckled honeycomb network of silicon atoms is a stable structure when it was firstly reported in $1994 .{ }^{21}$ For example, phonon dispersion calculations ${ }^{19}$ and first-principles calculations based on density functional theory (DFT) ${ }^{25}$ have clearly confirmed that freestanding silicene is stable and energetically degenerating. In addition, many studies on silicene are based on freestanding silicene including its hydrogenation. ${ }^{24,26,27}$ Therefore, freestanding silicene will be studied in this work.

Recently, several studies confirmed that silicene is a zero band gap semiconductor. ${ }^{20,24}$ This discovery brings the same challenge to silicene as for graphene that is controlling the band gap in order to vary the properties of silicene for future potential applications. With these expectations and the routes used for graphene, the hydrogenation of silicene has become a topic of great interest $\mathrm{t}^{28-35}$ and various breakthroughs in this area have been reported. When silicene is hydrogenated on a substrate, generally only the top side of Si atoms is hydrogenated, which makes the system show ferromagnetic properties. ${ }^{36}$ While half hydrogenated silicene without substrates turns out to be a 
ferromagnetic semiconductor with an indirect band gap (about $0.84 \mathrm{eV}$ ) based on generalized gradient approximation (GGA) calculations. ${ }^{26}$ While fully hydrogenated silicene, named silicane, generates about $2 \mathrm{eV}$ indirect band gap by local density approximation (LDA) calculations and becomes an insulator. ${ }^{24}$ Furthermore, it is also found that by applying an appropriate hydrogenation ratio the band structure of silicene can be tuned to produce metallic, semiconducting, or insulating properties. ${ }^{24,26}$ These results open a door to widen the applications of silicene-based devices.

However, according to the study of binding energy of low buckled silicene based on DFT-GGA calculations, ${ }^{37}$ it is found that the energy barrier of hydrogen atom adsorption on silicene is too high compared to that of gaseous hydrogen molecules, which indicates that the dissociative adsorption of hydrogen on silicene would be difficult due to the passive surface of silicene. The possibility of the dissociation can be understood by the energy barrier of this reaction, which is defined as the energy difference between the initial state and the highest energy along the minimum energy reaction paths (transition state). With high energy barrier, reactions would not take place effectively or not even occur. ${ }^{38,39}$ It is reported that the chemical reactions can be modified by an electric field, which would induce the electron redistribution, thus changing the chemical activity. ${ }^{40}$ For example, the dissociation activation energy of molecular oxygen on $\mathrm{Pt}(111)$ is changeable with an applied electric field. ${ }^{41}$ More recently, the energy barrier of the hydrogenation of graphene was reported to reduce to zero by applying an electric field with an appropriate intensity and the hydrogenation could take place smoothly and efficiently. ${ }^{42}$ For silicene, it is also found that an external electric field could control the band gap of silicene ${ }^{43}$ and at the silicene/(0001) ZnS interface. ${ }^{44}$ Therefore, an electric field is considered in this work to modify the electric properties and chemical activity of silicene as a catalyst.

In order to reduce the energy barrier of silicene hydrogenation, a perpendicular electric field is applied and its effect on hydrogenation is investigated through DFT calculations in this work. The hydrogenation of silicene can be understood through the calculation of the reaction pathway of one $\mathrm{H}_{2}$ molecule dissociative adsorption on silicene in the presence of the external electric field. The mechanism of the energy barrier reduction induced by the external electric field can be analyzed through partial density of states (PDOS). In addition, the effect of hydrogenation on the electronic properties of silicene is also discussed.

\section{Simulation methodology}

The DFT calculations are performed using the DMol3 code. The local density approximation (LDA) using the Perdew and Wang (PW91) functional is employed as the exchange-correlation functional. A double numerical plus polarization (DNP) basis set is used. The convergence tolerance of energy is taken as $10^{-5} \mathrm{Ha}(1 \mathrm{Ha}=27.21 \mathrm{eV})$. The maximum allowed force and displacement are $0.002 \mathrm{Ha}$ and $0.005 \AA$, respectively. Linear synchronous transition/quadratic synchronous transit (LST/QST) ${ }^{21}$ and nudged elastic band (NEB) ${ }^{45}$ tools in DMol3 are employed

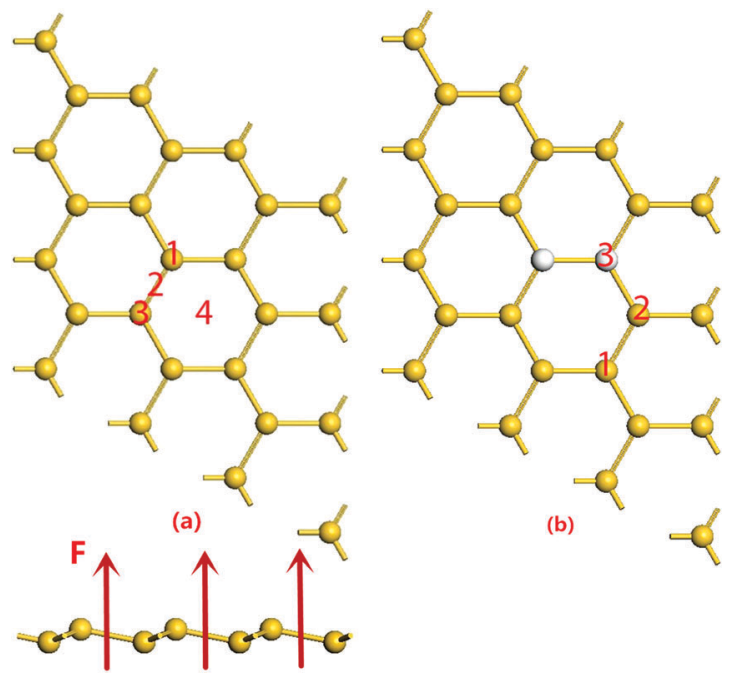

Fig. 1 (a) The four possible positions of $\mathrm{a}_{2}$ molecule physically adsorbed on silicene, (b) the three possible positions of the second $\mathrm{H}$ atom when $2 \mathrm{H}$ atoms chemically adsorbed on silicene. The direction of the positive electric field is denoted by arrows.

to investigate the minimum energy pathway for hydrogen dissociative adsorption on silicene. Three-dimensional periodic boundary conditions are taken in the simulation. A $3 \times 3 \times 1$ supercell is adopted for all the structures in the present simulations with a vacuum width of $18 \AA$ above the layer to minimize the interlayer interaction, as shown in Fig. 1. The $k$-point is set to $6 \times 6 \times 1$ and all atoms are allowed to relax in all the calculations.

The adsorption energy of an $\mathrm{H}_{2}$ molecule onto silicene, $E_{\mathrm{ad}}$, is defined as,

$$
E_{\text {ad }}=E_{\mathrm{H}_{2}+\text { silicene }}-\left(E_{\text {silicene }}+E_{\mathrm{H}_{2}}\right)
$$

where the subscripts $\mathrm{H}_{2}+$ silicene, silicene, and $\mathrm{H}_{2}$ denote the silicene with $\mathrm{H}_{2}$ molecules adsorbed, bare silicene, and $\mathrm{H}_{2}$ molecule, respectively. After hydrogenation, the binding energy of $\mathrm{H}$ atoms on silicene $E_{\mathrm{Si}-\mathrm{H}}$ can be determined as

$$
E_{\mathrm{Si}-\mathrm{H}}=E_{2 \mathrm{H}+\text { silicene }}-\left(E_{\text {silicene }}+2 E_{\mathrm{H}}\right)
$$

where subscripts $2 \mathrm{H}+$ silicene and $\mathrm{H}$ denote the silicene with $2 \mathrm{H}$ atoms chemically adsorbed and a free $\mathrm{H}$ atom, respectively.

\section{Results and discussion}

Before hydrogenation, the $\mathrm{H}_{2}$ molecule is weakly physically adsorbed on silicene. There are 4 possible adsorption positions as indicated in Fig. 1(a): positions 1 and 3 on the top of a silicon atom in the higher or lower plane of the buckled structure, position 2 over the middle of a $\mathrm{Si}-\mathrm{Si}$ bond or position 4 the center of a Si ring. In order to determine the most stable structure, we calculate all the four possible positions. The results in Table 1 show that the total energy and adsorption energy of a $\mathrm{H}_{2}$ molecule on silicene at positions 1-3 are almost the same but those at position 4 are lower, i.e. the $\mathrm{H}_{2}$ molecule is favorable to adsorb at the hollow site of the Si ring. This configuration is also 
Table 1 The total energy, binding energy, and the distance between the $\mathrm{H}_{2}$ molecule and the silicene layer of all the possible structures before (reactants) and after hydrogenation (products). For total energy, the energy of the most stable structure is set to be zero for comparison

\begin{tabular}{lccccrr}
\hline & \multicolumn{2}{c}{ Reactants } & & \multicolumn{3}{c}{ Products } \\
\hline Position & 1 & 2 & 3 & 4 & 1 & 2 \\
Total energy (eV) & 0.066 & 0.061 & 0.068 & 0 & 0.52 & 0.08 \\
Binding energy (eV) & -0.1 & -0.1 & -0.1 & -0.166 & -5.39 & -4.96 \\
$D_{\mathrm{H}-\mathrm{Si}}(\AA)$ & 3.2 & 3.1 & 3.6 & 2.45 & & -5.46
\end{tabular}

considered to be the reactant for the silicene hydrogenation reaction. Note that the distance between the $\mathrm{H}_{2}$ molecule and the silicene layer at position 4 is calculated to be $2.454 \AA$, which is shorter than that in the other structures due to the stronger binding energy. In the subsequent atomic hydrogen adsorption, there are three possible positions for the two hydrogen atoms, which are ortho, meta and para positions shown as the second $\mathrm{H}$ atom at positions 1-3 in Fig. 1(b). All the three possible configurations are calculated, and the corresponding results are listed in Table 1. It is found that the hydrogenated silicene with two hydrogen atoms in the ortho position, i.e. the second $\mathrm{H}$ atom at position 3 , has the lowest energy, the corresponding structure is shown in Fig. 2(b). In the hydrogenated silicene, the $\mathrm{Si}-\mathrm{H}$ bond length $l_{\mathrm{Si}-\mathrm{H}}$ is $1.50 \AA$ and the $\mathrm{Si}-\mathrm{Si}$ bond length $l_{\mathrm{Si}-\mathrm{Si}}$ is $2.355 \AA$, which agrees with reported results $l_{\mathrm{Si}-\mathrm{H}}=1.50 \AA, l_{\mathrm{Si}-\mathrm{Si}}=$ $2.36 \AA^{30}$ and $l_{\mathrm{Si}-\mathrm{H}}=1.519 \AA, l_{\mathrm{Si}-\mathrm{Si}}=2.359 \AA^{24}$ in fully hydrogenated silicene. In addition, the $\mathrm{Si}$ atoms bonded with the two $\mathrm{H}$ atoms moved up about $0.78 \AA$ towards the $\mathrm{H}$ atoms as shown in the side view of the hydrogenated silicene of Fig. 2(b). Fig. 2 also shows the band structures of silicene before and after hydrogenation. As shown in the bottom panel of Fig. 2(a), before hydrogenation, silicene is a zero bandgap semiconductor similar to graphene, and the physical adsorption of a $\mathrm{H}_{2}$ molecule does not change the band structure of silicene. However, after hydrogenation, the band gap of silicene opens about $110 \mathrm{meV}$ as shown in the bottom panel of Fig. 2(b), which is similar to the reported result when silicene is hydrogenated by $25 \%$ and band gap was calculated to be $148 \mathrm{meV} .{ }^{24}$ This means partial hydrogenation could also open a band gap in silicene, and its band gap depends on the ratio of hydrogenation, where half hydrogenated silicene turns out to be a semiconductor with a band gap about $0.84 \mathrm{eV}^{25}$ and fully hydrogenated silicene, named silicane, generates about 2 eV band gap. ${ }^{37}$

Due to the importance of hydrogenation of silicene, it is vital to understand the chemical reaction of the hydrogenation process. For $\mathrm{H}_{2}$ molecule dissociative adsorption on silicene, the structures of reactants and products are shown in Fig. 2(a) and (b). After LST/QST and NBE calculations, the energy minimum dissociative absorption pathway is shown in Fig. 3. From this figure, the dissociative adsorption reaction energy barrier $E_{\mathrm{bar}}=E_{\mathrm{TS}}-E_{\mathrm{IS}}$ is $1.75 \mathrm{eV}$ where $E_{\mathrm{TS}}$ and $E_{\mathrm{IS}}$ are respectively the energies of the transition state (TS) and the initial state (IS). Before the TS, the $\mathrm{H}_{2}$ molecule is dissociated into two free $\mathrm{H}$ atoms with a small energy barrier of around $0.1 \mathrm{eV}$, then one of the $\mathrm{H}$ atoms binds to a $\mathrm{Si}$ atom nearby at the top layer while the other $\mathrm{H}$ atom remains free, shown as the TS in Fig. 3. In addition, the Si atom that binds to the free $\mathrm{H}$ atom has also shifted upwards, but the two $\mathrm{H}$ atoms are

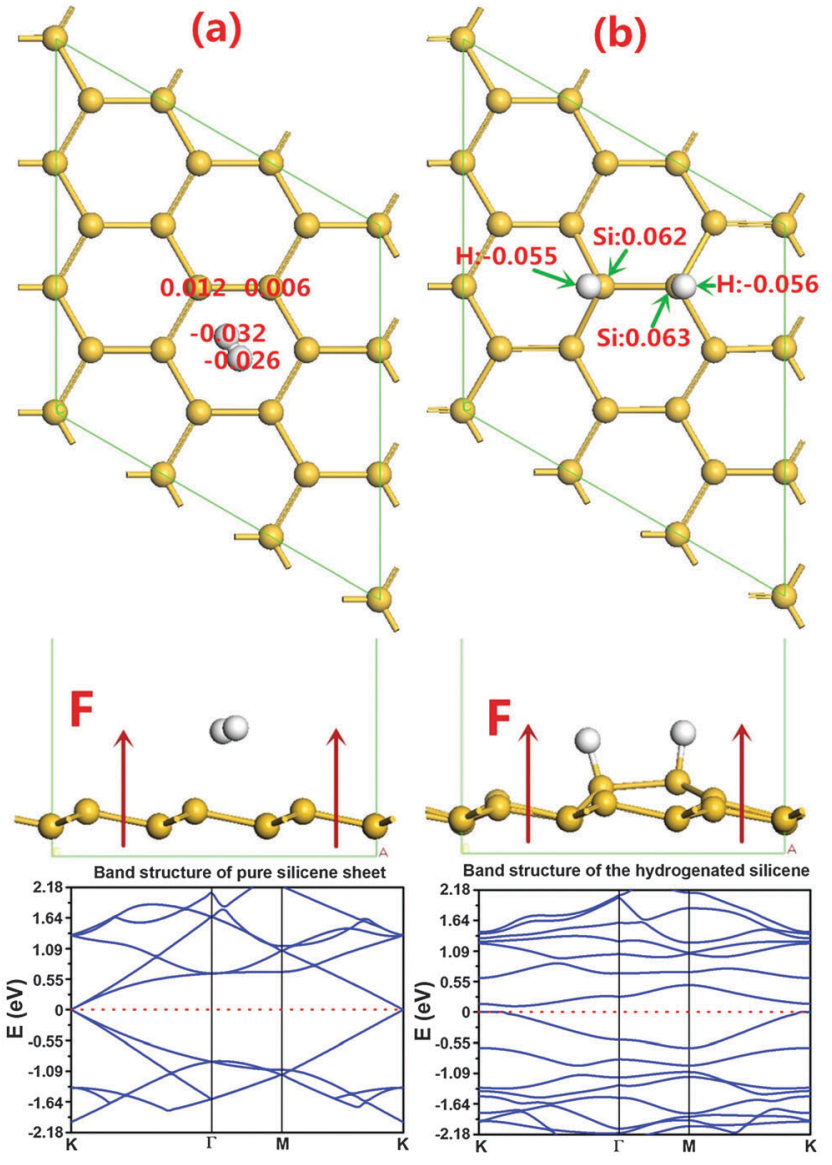

Fig. 2 The initial and final structures and the corresponding band structures of a $\mathrm{H}_{2}$ molecule on silicene before and after dissociative adsorption and atomic charge near the adsorbed hydrogen. The numbers at the top panel of the figure denote the atomic charge of the atoms.

still close to each other with strong interaction, which prevents the binding between the free $\mathrm{H}$ atom and the $\mathrm{Si}$ atom. At the final state FS, the distance of the two $\mathrm{H}$ atoms increases, and thus the other free $\mathrm{H}$ atom also binds with the nearest $\mathrm{Si}$ atom as shown in Fig. 3. Therefore, this reaction can be separated into two steps: the $\mathrm{H}_{2}$ molecule is first dissociated into two $\mathrm{H}$ atoms, and then one of them binds with a Si atom nearby while the other one is free; at the second step, the two $\mathrm{H}$ atoms adjust their positions and the other $\mathrm{H}$ atom also binds with its nearest $\mathrm{Si}$ atom. Although this reaction releases an energy of about $0.25 \mathrm{eV}$ in total (total energy of the final state $E_{\mathrm{FS}}-E_{\mathrm{IS}}$ ), there is a high energy barrier of $\sim 1.75 \mathrm{eV}$ for the first step. Consequently, the first step becomes a rate-limiting step because of high energy needed. 


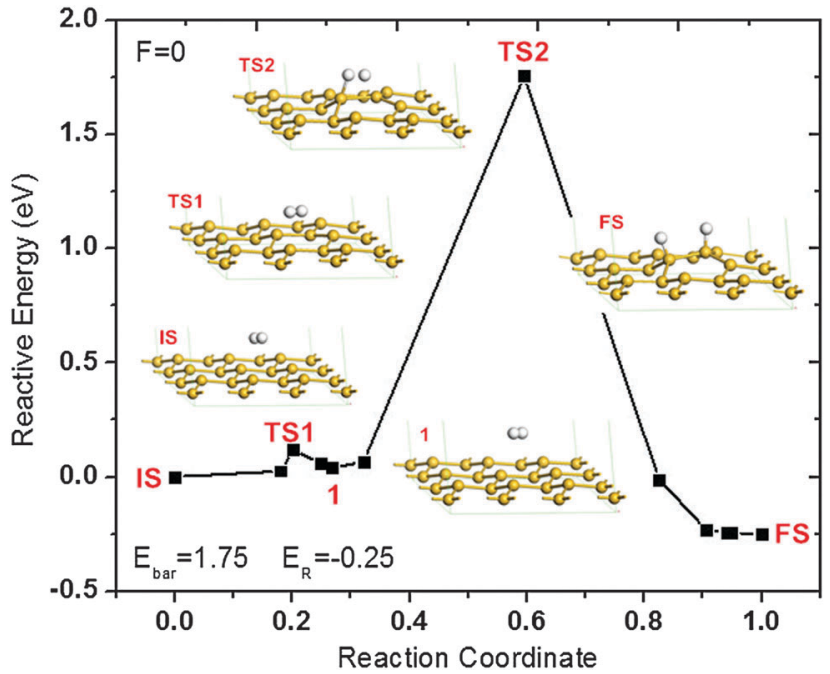

Fig. 3 The reaction pathway of the dissociative adsorption of a $\mathrm{H}_{2}$ molecule on silicene. The energy of the reactant IS is taken to be zero. IS, TS and FS denote initial structure, transition structure, and final structure, respectively. $E_{\mathrm{bar}}$ is the energy barrier and $E_{\mathrm{R}}$ is the reaction energy.

It is reported that a reaction is difficult to perform at room temperature if the reaction energy barrier is higher than $0.75 \mathrm{eV}{ }^{46}$ Due to the high energy barrier of $1.75 \mathrm{eV}$ for this hydrogenation process, the reaction is difficult to perform at room temperature, hence high reaction temperature or other external energy source is required. Therefore, it is desirable to reduce the hydrogenation energy barrier. Applying an external electric field is an alternative way to alter the electronic distribution of silicene, thus changing its chemical potential. In addition, the electric field is found to be a catalyst to reduce the reaction barriers for the dissociative adsorption of $\mathrm{H}_{2} \mathrm{O}$ on graphene, ${ }^{47}$ and $\mathrm{H}_{2}$ on graphene as well. ${ }^{42}$ Therefore, an external electric field is considered here to investigate the possibility of reducing the hydrogenation energy barrier of silicene.

To investigate the effect of an electric field, electric fields with different directions and intensities are considered, the electric field along the arrows in Fig. 1 is defined as the positive electric field. It is found that the positive electric field can reduce the energy barrier for the hydrogenation reaction, while the negative electric field has an opposite effect on the hydrogenation reaction, increasing the energy barrier. Therefore, only the result of energy barrier under positive electric fields is shown in Fig. 4. It is clearly shown that the electric field can reduce the energy barrier of $\mathrm{H}_{2}$ molecule dissociative adsorption on silicene remarkably. The energy barrier decreases with the intensity of the electric field increasing. Note that $E_{\mathrm{bar}}$ is lower than $0.75 \mathrm{eV}$ when $F$ is above 0.05 a.u. i.e., the $\mathrm{H}_{2}$ molecule can be dissociated and adsorbed on silicene automatically at room temperature in the presence of the electric field of 0.05 a.u. If the intensity of the electric field is increased further up to 0.06 a.u., the barrier can be reduced further. However, when $F \geq 0.06$ a.u., the structure of silicene would be destroyed, which should be avoided in applications.

To better understand the effect of electric fields, the hydrogenation pathways of silicene under different electric fields are

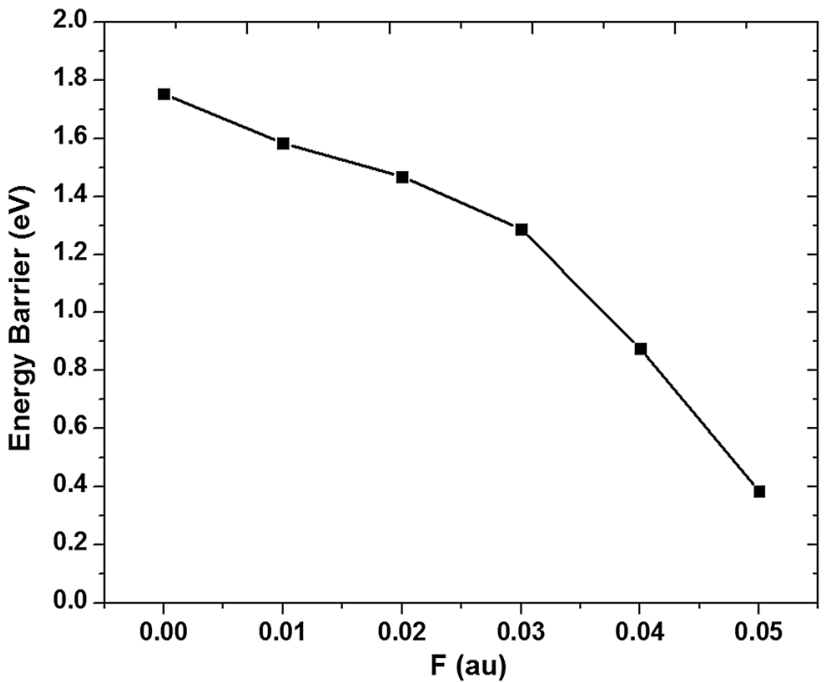

Fig. 4 Energy barrier of the dissociative adsorption of a $\mathrm{H}_{2}$ molecule on silicene under the positive perpendicular electric fields. The units of $E_{\mathrm{bar}}$ and $F$ are eV and a.u., respectively.

shown in Fig. 5. As shown in the figures, the configurations before and after hydrogenation reconstructed as expected when the electric field is strong enough. When $F \geq 0.03$ a.u., after geometry optimization, the reactants transferred from the IS to an energy minimum state, state 1 . At state 1 , the $\mathrm{H}_{2}$ molecule slightly adjusts its position and orientation over the silicene layer. Then the $\mathrm{H}_{2}$ molecule is dissociated into $2 \mathrm{H}$ atoms at the $\mathrm{TS}$, while one of the $\mathrm{H}$ atoms binds with a $\mathrm{Si}$ atom in the upper layer for the cases in Fig. 5(a)-(c). When $F=0.05$ a.u. as shown in Fig. 5(d), different from other cases, the two $\mathrm{H}$ atoms bind to the same Si atom at the TS. This special binding is probably caused by the strongly upward force on the positively charged silicon atom and the downward force on the negatively charged hydrogen atom under the positive electric field. Similar to the case without an electric field, the two $\mathrm{H}$ atoms are still close to each other with relatively strong interaction. After the binding of the second $\mathrm{H}$ atom to a $\mathrm{Si}$ atom, the $\mathrm{H}-\mathrm{H}$ interaction is reduced and their distance is increased remarkably as shown in state 2 of Fig. 5(d). As expected, the structure of the hydrogenated silicene would be reconstructed as well in the presence of an electric field, which is shown as the state 2 in Fig. 5 except the case at $F=0.01$ a.u., which is considered to be too weak to change the structure. Therefore, under a strong positive electric field, the reaction would stop at state 2 . Once the electric field is removed, the structure of state 2 would reconstruct into the structure of the FS. In addition, the reaction energy $E_{\mathrm{R}}$ decreases as $F$ increases, which would further benefit the hydrogenation process. Therefore, silicene can be hydrogenated spontaneously under the electric field of 0.05 a.u. with a low energy barrier of $0.38 \mathrm{eV}$.

As mentioned above, we also investigated this reaction in the presence of a negative electric field, but it is found that the energy barrier increases to $1.92 \mathrm{eV}$ under an electric field of -0.02 a.u. If the electric field is further increased, the silicene 

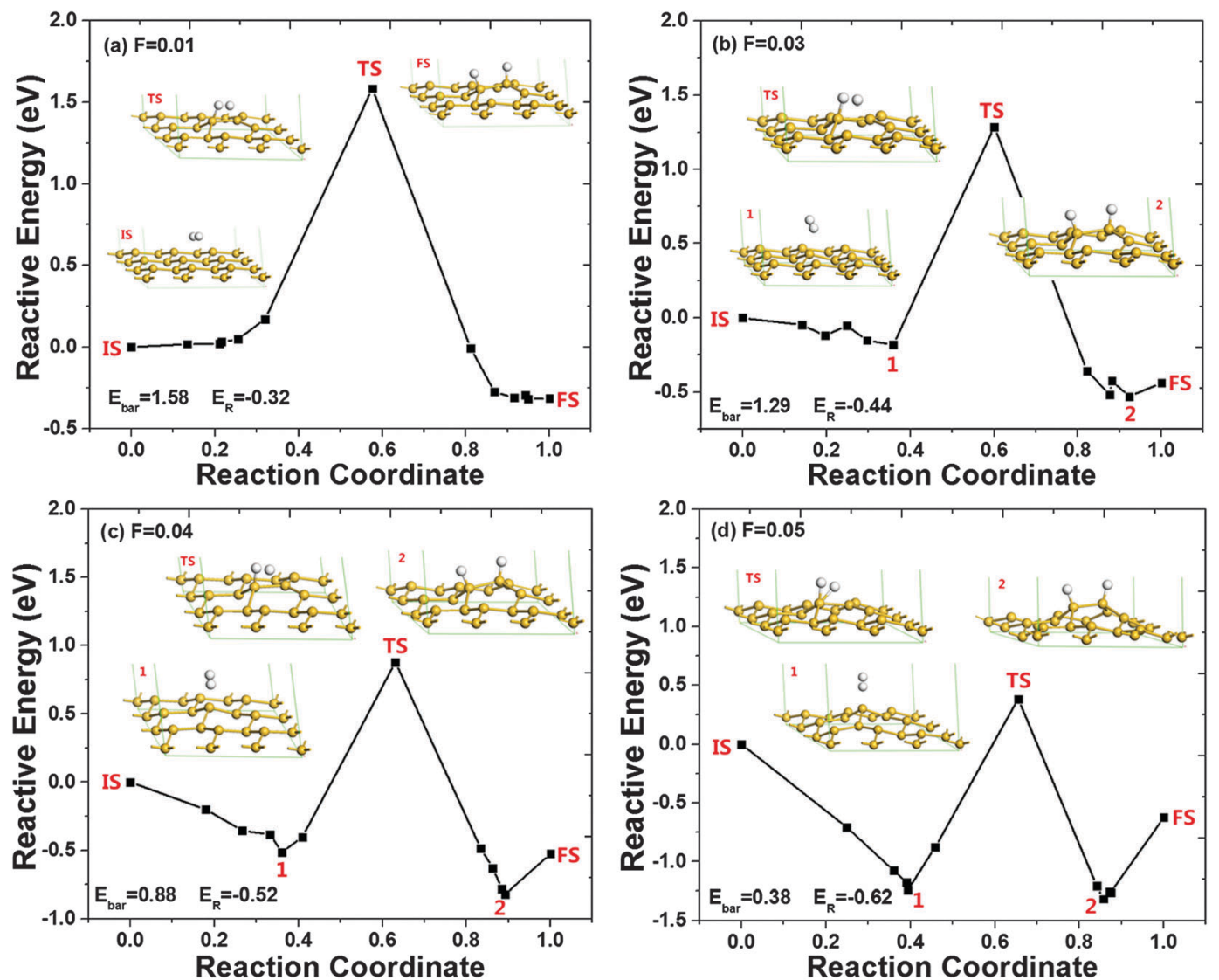

Fig. 5 The pathway of dissociative adsorption of a $\mathrm{H}_{2}$ molecule on silicene in the presence of positive electric fields. The transition structure and energy minimum states 1 and 2 are represented by TS, and 1 and 2 respectively. The initial structure IS and the final structure FS are even shown in Fig. 1 . The energy in the IS is set to be zero. The $E_{\mathrm{bar}}$ and $E_{\mathrm{R}}$ are the energy barrier and the reaction energy, respectively, and their units are in eV.

structure would be destroyed, which is similar to the investigation of the effects of an electronic field on the atomic structure of the graphene $/ \alpha-\mathrm{SiO}_{2}(0001)$ interface ${ }^{48}$ where the structure of graphene was destroyed and covalent bonds between graphene and the $\mathrm{SiO}_{2}$ substrate are formed under a strong negative electric field. It is known from Fig. 2 that $\mathrm{H}$ atoms are negatively charged and the $\mathrm{Si}$ atoms bonded to the $\mathrm{H}$ atoms are positively charged. In the presence of a negative electric field, the distance between $\mathrm{Si}$ and $\mathrm{H}$ atoms would increase due to the upward force on $\mathrm{H}$ atoms while downward force on $\mathrm{Si}$ atoms. Therefore, the negative electric field prevents the formation of covalent bonds between $\mathrm{Si}$ and $\mathrm{H}$ atoms and results in higher hydrogenation energy barrier. In contrast, in the presence of a positive electric field, the negatively charged $\mathrm{H}$ atoms are attracted downward and the positively charged $\mathrm{Si}$ atoms are pushed upward by the electric field, which facilitates the formation of $\mathrm{Si}-\mathrm{H}$ bonds. Therefore, the positive electric field can reduce the hydrogenation energy barrier of silicene.

The effect of electric fields on hydrogenation energy barrier of silicene can also be understood through analyzing PDOS of the configuration at TS under different electric fields as shown in Fig. 6. The bands of the s orbital of the two $\mathrm{H}$ atoms and the $\mathrm{p}$ orbital of the two corresponding $\mathrm{Si}$ atoms binding with the two
$\mathrm{H}$ atoms at the TS are provided. It is clear that the interaction of the lowest $\mathrm{Si}-\mathrm{H}$ interaction band (the weight of the overlap between the bands of the s orbital of $\mathrm{H}$ and the porbital of $\mathrm{Si}$ ) between -0.2 and $-0.4 \mathrm{eV}$ is enhanced when $F$ increases, while the interaction band near the Fermi level does not change much under positive electric fields. Under a negative electric field, the lowest band is weakened and the band near the Fermi level is enhanced. It is known that the interaction of the lowest band determines the interaction of $\mathrm{Si}-\mathrm{H}$, and thus determines the energy barrier. Therefore, when $F=$ 0.05 a.u., the $\mathrm{Si}-\mathrm{H}$ interaction is the strongest, thus it is easier for the $\mathrm{H}_{2}$ molecule to dissociate and form a covalent bond with $\mathrm{Si}$, and the corresponding hydrogenation barrier is the lowest, which agrees with the result in Fig. 5.

As mentioned above, the electronic properties of silicene could be tuned by the ratio of hydrogenation. ${ }^{24,26,27}$ For the hydrogenation of graphene, it is reported that a large ratio of hydrogenation is difficult to achieve due to $\mathrm{H}$ frustration based on DFT and molecular dynamic (MD) calculations ${ }^{49}$ and experiments also showed that extensive hydrogenation is hard to achieve. ${ }^{50}$ Nevertheless, silicene has a buckled structure unlike the planar structure of graphene because sp3 hybridization is more stable than sp2 hybridization of silicon. ${ }^{24}$ This may cause a difference in the hydrogenation between silicene and 

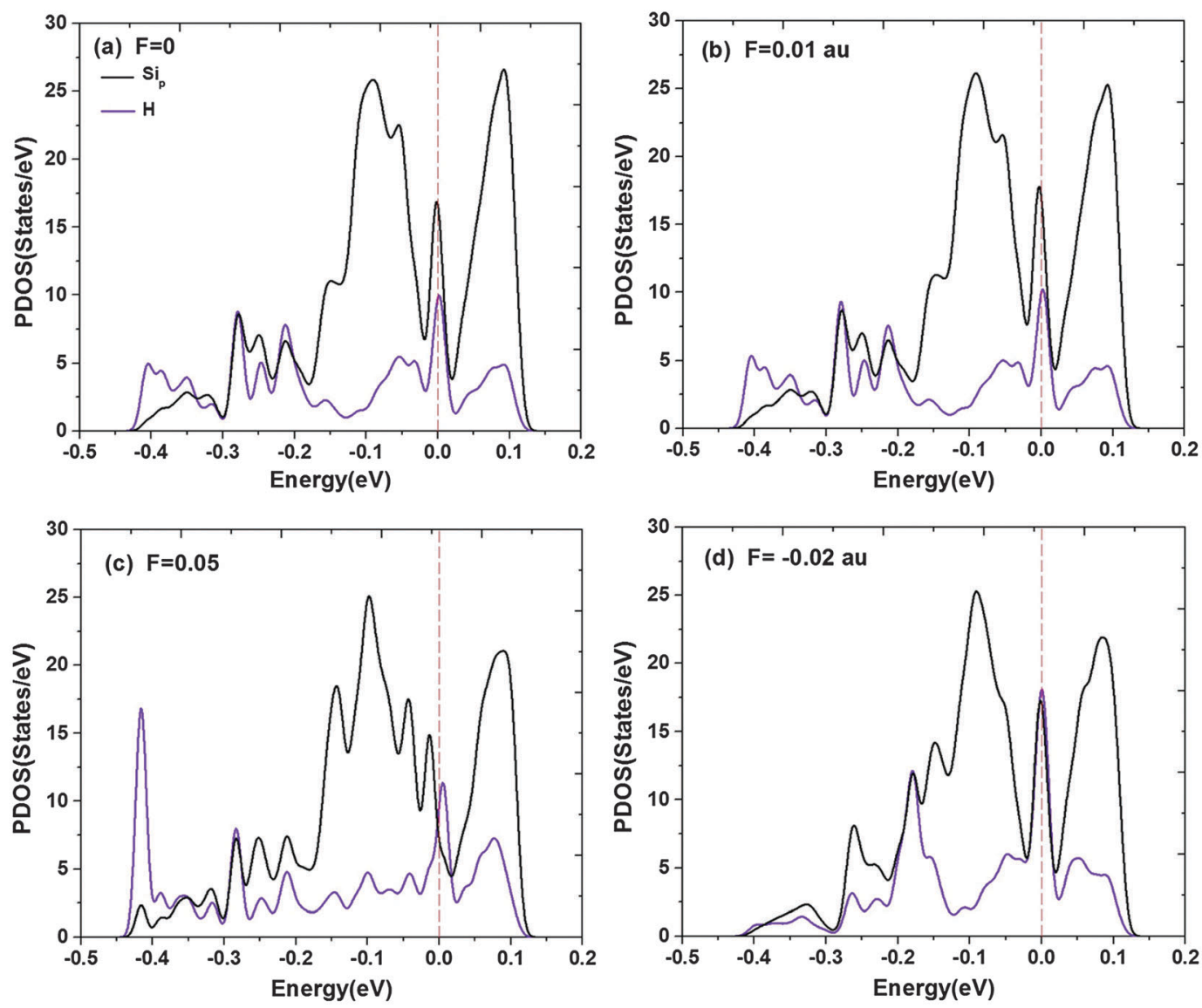

Fig. 6 The PDOS analysis of the two H atoms and the two corresponding Si atoms at the TS under different electric fields. The black curve is the PDOS of the two silicon atoms and the purple one indicates that of the two hydrogen atoms. The red dashed line stands for the Fermi level.

graphene. In addition, hydrogenation is believed to be easier near defects, ${ }^{51}$ which are unavoidable in actual situations. Therefore, different from graphene, the large ratio of hydrogenation of silicene may be possible, which will be studied in our following studies.

\section{Conclusion}

The dissociative adsorption of $\mathrm{a}_{2}$ molecule on silicene in the presence of different electric fields is investigated using DFT calculations. It is found that the energy barrier of dissociative adsorption of $\mathrm{a}_{2}$ molecule on silicene can be reduced by applying a positive perpendicular electric field, which can act as a catalyst to facilitate the silicene hydrogenation reaction, while negative electric fields have a converse effect on this reaction to increase the reaction energy barrier. In addition, the energy barrier decreases as the electric field increases. When the intensity of the positive electric field is 0.05 a.u. or larger, the barrier is lower than $0.75 \mathrm{eV}$, and the hydrogenation would happen smoothly and efficiently at room temperature. Therefore, we propose an alternative method to hydrogenate silicene, which is essential to open its band gap for its application in electronic devices.

\section{Acknowledgements}

We acknowledge the financial support from the Chancellor's Research Fellowship Program of the University of Technology, Sydney. This research was supported by the National Computational Infrastructure (NCI) through the merit allocation scheme and used NCI resources and facilities in Canberra, Australia.

\section{References}

1 K. S. Novoselov, A. K. Geim, S. V. Morozov, D. Jiang, M. I. Katsnelson, I. V. Grigorieva, S. V. Dubonos and A. A. Firsov, Nature, 2005, 438, 197.

2 M. I. Katsnelson, K. S. Novoselov and A. K. Geim, Nat. Phys., 2006, 2, 620 .

3 A. K. Geim and K. S. Novoselov, Nat. Mater., 2007, 6, 183.

4 G. Giovannetti, P. A. Khomyakov and G. Brocks, Phys. Rev. B: Condens. Matter Mater. Phys., 2007, 76, 073103.

5 S. Y. Kwon, C. V. Ciobanu and V. B. Shenoy, Nano Lett., 2009, 9, 3985.

6 T. Ohta, A. Bostwick, T. Seyller, K. Horn and E. Rotenberg, Science, 2006, 313, 951.

7 M. Y. Han, B. Ozyilmaz, Y. Zhang and P. Kim, Phys. Rev. Lett., 2007, 98, 206805. 
8 J. O. Sofo, A. S. Chaudhari and G. D. Barber, Phys. Rev. B: Condens. Matter Mater. Phys., 2007, 75, 153401.

9 D. C. Elias, R. R. Nair, T. M. G. Mohiuddin, S. V. Morozov, P. Blake, M. P. Halsall, A. C. Ferrari, D. W. Boukhvalov, M. I. Katsnelson, A. K. Geim and K. S. Novoselov, Science, 2009, 323, 610.

10 B. S. Pujari, S. Gusarov, M. Brett and A. Kovalenko, Phys. Rev. B: Condens. Matter Mater. Phys., 2011, 84, 041402.

11 C. Srinivasan and R. Saraswathi, Curr. Sci., 2009, 97, 302.

12 L. B. Drissi, E. H. Saidi, M. Bousmina and O. Fassi-Fehri, J. Phys.: Condens. Matter, 2012, 24, 485502.

13 J. Zhou, Q. Wang, Q. Sun, X. S. Chen, Y. Kawazoe and P. Jena, Nano Lett., 2009, 9, 3867.

14 D. W. Boukhvalov, M. I. Katsnelson and A. I. Lichtenstein, Phys. Rev. B: Condens. Matter Mater. Phys., 2008, 77, 035427.

15 J. Li, T. Furuta, H. Goto, T. Ohashi, Y. Fujiwara and S. Yip, J. Chem. Phys., 2003, 119, 2376.

16 B. Lalmi, H. Oughaddou, H. Enriquez, A. Kara, S. Vizzini, B. Ealet and B. Aufray, Appl. Phys. Lett., 2010, 97, 223109.

17 B. Aufray, A. Kara, S. Vizzini, H. Oughaddou, C. Léandri, B. Ealet and G. Le Lay, Appl. Phys. Lett., 2010, 96, 183102.

18 S. Lebégue and O. Eriksson, Phys. Rev. B: Condens. Matter Mater. Phys., 2009, 79, 115409.

19 S. Cahangirov, M. Topsakal, E. Akturk, H. Sahin and S. Ciraci, Phys. Rev. Lett., 2009, 102, 236804.

20 M. Houssa, E. Scalise, K. Sankaran, G. Pourtois, V. V. Afanasev and A. Stesmans, Appl. Phys. Lett., 2011, 98, 223107.

21 K. Takeda and K. Shiraishi, Phys. Rev. B: Condens. Matter Mater. Phys., 1994, 50, 14916.

22 H. Nakano, T. Mitsuoka, M. Harada, K. Horibuchi, H. Nozaki, N. Takahashi, T. Nonaka, Y. Seno and H. Nakamura, Angew. Chem., 2006, 118, 6451.

23 B. Lalmi, H. Oughaddou, H. Enriquez, A. Kara, S. Vizzini, B. Ealet and B. Aufray, Appl. Phys. Lett., 2010, 97, 223109.

24 T. H. Osborn, A. A. Farajian, O. V. Pupysheva, R. S. Aga and L. C. Lew Yan Voon, Chem. Phys. Lett., 2011, 511, 101.

25 M. Houssa, G. Pourtois, V. V. Afanasev and A. Stesmans, Appl. Phys. Lett., 2010, 97, 112106.

26 P. Zhang, X. D. Li, C. H. Hu, S. Q. Wu and Z. Z. Zhu, Phys. Lett. A, 2012, 376, 1230.

27 L. C. Lew Yan Voon, E. Sandberg, R. S. Aga and A. A. Farajian, Appl. Phys. Lett., 2010, 97, 163114.

28 M. R. Linford and C. E. D. Chidsey, J. Am. Chem. Soc., 1993, 115, 12631.
29 G. P. Lopinski, D. D. M. Wayner and R. A. Wolkow, Nature, 2000, 406, 48.

30 Md. Z. Hosssain, H. S. Kato and M. Kawai, J. Am. Chem. Soc., 2005, 127, 15030.

31 Md. Z. Hosssain, H. S. Kato and M. Kawai, J. Phys. Chem. B, 2005, 109, 23129.

32 J. K. Kang and C. B. J. Musgrave, J. Chem. Phys., 2002, 116, 9907.

33 N. Takeuchi, Y. Kanai and A. Selloni, J. Am. Chem. Soc., 2004, 126, 15890.

34 N. Takeuchi and A. Selloni, J. Phys. Chem. B, 2005, 109, 11967.

35 N. Takeuchi, Y. Kanai and A. Selloni, J. Phys. Chem. C, 2010, 114, 3981.

36 F. B. Zheng and C. W. Zhang, Nanoscale Res. Lett., 2012, $7,422$.

37 A. Kara, H. Enriquez, A. P. Seitsonen, L. C. Lew Yan Voon, S. Vizzini, B. Aufray and H. Oughaddou, Surf. Sci. Rep., 2012, $67,1$.

38 P. R. Pereda and N. Takeuchi, J. Chem. Phys., 2013, 138, 194702.

39 N. Takeuchi and A. Selloni, J. Phys. Chem. B, 2005, 109, 11967. 40 J. S. McEwen, P. Gaspard, F. Mittendorfer, T. Visart de Bocarmé and N. Kruse, Chem. Phys. Lett., 2008, 452, 133.

41 M. P. Hyman and J. W. Medlin, J. Phys. Chem. B, 2005, 109, 6304.

42 Z. M. Ao and F. M. Peeters, Appl. Phys. Lett., 2010, 96, 253106.

43 G. Rahman, Europhys. Lett., 2014, 105, 37012.

44 M. Houssa, B. van den Broek, E. Scalise, G. Pourtois, V. V. Afanasev and A. Stesmans, Phys. Chem. Chem. Phys., 2013, 15, 3702.

45 G. Henkelman and H. Jonsson, J. Chem. Phys., 2000, 113, 9978.

46 C. Shang and Z. P. Liu, J. Am. Chem. Soc., 2011, 133, 9938.

47 Q. G. Jiang, Z. M. Ao, D. W. Chu and Q. Jiang, J. Phys. Chem. C, 2012, 116, 19321.

48 Z. M. Ao, W. T. Zheng and Q. Jiang, Nanotechnology, 2008, 19, 275710.

49 M. Z. S. Flores, P. A. S. Autreto, S. B. Legoas and D. S. Galvao, Nanotechnology, 2009, 20, 465704.

50 H. L. Poh, F. Sanek, Z. Sofer and M. Pumera, Nanoscale, 2012, 4, 7006.

51 Q. G. Jiang, Z. M. Ao, W. T. Zheng, S. Li and Q. Jiang, Phys. Chem. Chem. Phys., 2013, 15, 21016. 